\title{
Capsaicin Responses in Heat-Sensitive and Heat-Insensitive A-Fiber Nociceptors
}

\author{
Matthias Ringkamp, ${ }^{1}$ Yuan B. Peng, ${ }^{1}$ Gang Wu, ${ }^{1}$ Timothy V. Hartke, ${ }^{1}$ James N. Campbell, ${ }^{1,2}$ and \\ Richard A. Meyer ${ }^{1,2}$ \\ ${ }^{1}$ Department of Neurosurgery, School of Medicine, Johns Hopkins University, Baltimore, Maryland 21287, and 2Johns \\ Hopkins Applied Physics Laboratory, Laurel, Maryland 20723
}

The recently cloned vanilloid receptor (VR1) is postulated to account for heat and capsaicin sensitivity in unmyelinated afferents. We sought to determine whether heat and capsaicin sensitivity also coexist in myelinated nociceptive afferents. Action potential (AP) activity was recorded from single A-fiber nociceptors that innervated the hairy skin in monkey. Before intradermal injection of capsaicin $(10 \mu \mathrm{g} / 10 \mu \mathrm{l})$ into the receptive field, nociceptors were classified as heat-sensitive (threshold, $\leq 53^{\circ} \mathrm{C}, 1 \mathrm{sec}$ ) or heat-insensitive afferents and as mechanically sensitive (von Frey threshold, $<6$ bar) or mechanically insensitive afferents. All heat-sensitive afferents $(n=16)$ were insensitive to mechanical stimuli but responded to the intradermal injection of capsaicin (69 \pm 7 APs in 10 min). Responsiveness to mechanical stimuli, thermal stimuli, and capsaicin varied in their receptive fields; the majority of receptive field sites (24 of 36) were responsive to only one or two stimulus modal-

Intradermal injection of capsaicin in humans produces intense pain and secondary hyperalgesia. Intradermal injection of capsaicin weakly activates conventional C-fiber nociceptors (Baumann et al., 1991) but produces a vigorous response in a subtype of $\mathrm{C}$-fiber nociceptors that are insensitive to mechanical stimuli (Schmelz et al., 2000). However, little is known about the responses of A-fiber nociceptors to capsaicin, and therefore the role of A-fiber nociceptors in capsaicin-evoked pain and hyperalgesia is unknown.

A-fiber nociceptive afferents in primate have been classified into three types based on their responsiveness to heat stimuli (Treede et al., 1995, 1998). Type I afferents are relatively insensitive to heat stimuli but respond with a long latency to intense, long-duration heat stimuli (e.g., $53^{\circ} \mathrm{C}, 30 \mathrm{sec}$ ). Type II afferents are sensitive to heat (threshold $\sim 47^{\circ} \mathrm{C}, 1 \mathrm{sec}$ ) and respond briskly (latency, $\leq 200 \mathrm{msec}$ ) to intense heat. High-threshold mechanoreceptive nociceptors (HTMs) are unresponsive to heat stimuli. The relative response of these different classes of A-fiber nociceptors to capsaicin is unknown.

For unmyelinated nociceptors, heat and capsaicin sensitivity are thought to coexist and to be encoded, at least in part, by the recently cloned vanilloid receptor 1 (VR1) (Caterina et al., 1997). It appears that heat and capsaicin sensitivity may also coexist in

Received Dec. 27, 2000; revised March 12, 2001; accepted March 26, 2001.

This research was supported by National Institutes of Health Grant NS 14447. We appreciate the technical assistance of Sylvia Horasek.

Correspondence should be addressed to Richard A. Meyer, Department of Neurosurgery, School of Medicine, Johns Hopkins University, Meyer 5-109, 600 North Wolfe Street, Baltimore, MD 21287. E-mail: rmeyer@jhmi.edu.

Copyright (C) 2001 Society for Neuroscience 0270-6474/01/214460-09\$15.00/0 ities, whereas only eight sites responded to all three modalities. For most heat-insensitive afferents, the activity induced by the capsaicin injection did not exceed the activity induced by needle insertion alone. However, the largest response to capsaicin (314 \pm 98 APs in $10 \mathrm{~min}$ ) was observed for five afferents that were insensitive to heat as well as mechanical stimuli and therefore may be classified as cutaneous chemoreceptors. These results suggest that A-fiber nociceptors play a role in the pain and hyperalgesia associated with capsaicin injection. Our finding that a subgroup of capsaicin-sensitive A-fiber nociceptors are insensitive to heat predicts the existence of heatinsensitive capsaicin receptors.

Key words: capsaicin; myelinated nociceptors; heat sensitivity; primate; monkey; hairy skin; receptive field; mechanically insensitive afferents some A-fiber nociceptors. First pain to heat is mediated by type II A-fiber nociceptors (Campbell and LaMotte, 1983). Because first pain to heat is lost after topical application of capsaicin, type II A-fiber nociceptors must have capsaicin sensitivity. However, the VR1 receptor is not found in myelinated afferents. Thus, a similar receptor likely accounts for heat sensitivity in type II A-fiber nociceptors.

The recently cloned vanilloid receptor-like protein (VRL1) receptor has a high heat threshold and is found in large dorsal root ganglia neurons. The VRL1 receptor is proposed to be responsible for heat transduction of type I A-fiber nociceptors (Caterina et al., 1999). Because the VRL1 receptor does not respond to capsaicin, type I A-fiber nociceptors are thought to be insensitive to capsaicin.

To elucidate further the relationship between heat and capsaicin sensitivity in nociceptive neurons and to assess the potential role of A-fiber nociceptors in capsaicin-evoked pain, we investigated in the present study the capsaicin responses of A-fiber nociceptive afferents. Because type I heat-insensitive and type II heat-sensitive A-fiber nociceptors have been found in nonhuman primates, studies were conducted in monkeys.

\section{MATERIALS AND METHODS}

General procedures. Monkeys (Macaca fascicularis and Macaca mulatta) were initially sedated with ketamine $(10 \mathrm{mg} / \mathrm{kg})$ followed by an intravenous bolus injection of pentobarbital $(7 \mathrm{mg} / \mathrm{kg})$ to achieve anesthesia. A continuous intravenous infusion of pentobarbital $\left(3-6 \mathrm{mg} \cdot \mathrm{kg}^{-1} \cdot \mathrm{hr}^{-1}\right.$ ) or, alternatively, a mixture of pentobarbital $\left(3 \mathrm{mg} \cdot \mathrm{kg}^{-1} \cdot \mathrm{hr}^{-1}\right)$ and morphine sulfate $\left(0.5 \mathrm{mg} \cdot \mathrm{kg}^{-1} \cdot \mathrm{hr}^{-1}\right)$ was started. Animals were hydrated with a continuous infusion of $5 \%$ dextrose in physiological saline solution $\left(4-6 \mathrm{mg} \cdot \mathrm{kg}^{-1} \cdot \mathrm{hr}^{-1}\right)$. To prevent bacterial infection, a prophy- 
lactic intramuscular injection of penicillin $\mathrm{G}$ was given $(300,000 \mathrm{U})$. Heart rate was continuously monitored by an electrocardiogram and used as an indicator of adequate anesthesia. Heart rate was kept within $10 \%$ of the initial baseline rate before any manipulations. Upon an increase in heart rate during or after a noxious intervention, a bolus of anesthetic was given to secure an adequate level of anesthesia. Muscle relaxation was induced by an intravenous dose of pancuronium bromide $(0.1 \mathrm{mg} / \mathrm{kg})$ and maintained by supplemental doses every $2-3 \mathrm{hr}$. Animals were intubated and ventilated with expired $\mathrm{pCO}_{2}$ adjusted to $40 \mathrm{mmHg}$. Animal core temperature was measured by a rectal probe and maintained at $38^{\circ} \mathrm{C}$ using feedback-controlled, water-perfused heating pads. All procedures were approved by the Johns Hopkins Animal Care and Use Committee.

Recordings were made from nerves innervating hairy skin, including the saphenous $(n=14)$, superficial peroneal $(n=6)$, sural $(n=2)$, superficial radial $(n=17)$, and medial antebrachial cutaneous nerves $(n=6)$. Teased-fiber recording techniques were used as described in detail previously (Campbell and Meyer, 1983). Briefly, the cutaneous nerve of interest was isolated from the surrounding connective tissue under a dissecting microscope. The skin around the incision was used to form a pool by suturing the edges to a metal ring. The pool was filled with paraffin oil. A splitting platform, which also served as the ground electrode, was placed underneath the nerve, and a small silver wire, which served as the recording electrode, was positioned above the splitting platform. Small bundles were cut from the nerve, and their distal stumps were teased into small filaments from which single fibers could be recorded. Signals were differentially amplified, filtered, and displayed on an oscilloscope (see also data collection). Between the splitting platform and the distal edge of the pool, a tripolar electrode was positioned on the nerve with the outer electrodes serving as anodes. This distal stimulation electrode was used to deliver electrical pulses of variable strength to the nerve to identify the fibers on the recording electrode and to determine their conduction latency. To calculate the conduction velocity, the conduction length between the recording electrode and the nerve stimulation electrode (typically $3-4 \mathrm{~cm}$ ) was measured and divided by the conduction latency obtained at an intensity three times above threshold for excitation. Fibers with a conduction velocity $>2 \mathrm{~m} / \mathrm{sec}$ as determined from the nerve stimulation electrode were regarded as A-fibers (Treede et al., 1998).

Mapping the receptive field. The cutaneous receptive field of the afferent under study was localized by gently squeezing the skin in the innervation territory of the respective nerve. The mechanoreceptive field was mapped using a von Frey hair. For mechanically sensitive afferents (MSAs), an 8.6 bar von Frey hair was used, whereas for mechanically insensitive afferents (MIAs) (von Frey threshold, $>6$ bar) (Meyer et al., 1991), a 15 bar von Frey hair was used. Mechanically sensitive spots were marked on the skin with colored ink. After mapping, the mechanical threshold of the afferent fiber was determined by applying a series of von Frey hairs of increasing strength. The weakest von Frey hair that induced a reliable activation of the afferent was defined as mechanical threshold.

If the receptive field of the afferent under study could not be localized by gently squeezing the skin, a previously described electrical search paradigm was used (Meyer et al., 1991). Briefly, electrical stimuli (up to $150 \mathrm{~V}, 2$ msec duration) were delivered transcutaneously using a salinesoaked cotton swab as a monopolar cathodic search electrode. A needle inserted into remote distal tissue served as the return electrode. Beginning at the distal edge of the recording pool, the cotton swab electrode was moved distally along the anticipated course of the peripheral nerve, thus increasing the conduction length and conduction latency of the afferent fiber under study. Increases in conduction latency were monotonic and continuous until the electroreceptive field was encountered in which the electrical threshold to activate the afferent reached a minimum and the conduction latency decreased in discrete steps ("hopping") with increasing stimulus intensity. The decrease in electrical threshold to a minimum is thought to reflect activation of superficial axon terminals, and hopping of the conduction latency may reflect activation of different branches in the peripheral cutaneous arborization of the nerve fiber (for details, see Peng et al., 1999). Test locations were defined as part of the electroreceptive field whenever discrete steps in latency were observed when the stimulation intensity was varied. To minimize current spread across the receptive field, which might compromise precise mapping of the electroreceptive field, the position of the return electrode was changed during the mapping so that it was always on the same side of the receptive field as the test electrode. Electroreceptive spots were marked with colored ink on the skin. The distance between the recording electrode and the receptive field was measured along the assumed course of the nerve. The conduction latency from the peripheral receptive field was determined at three times above threshold and used to calculate the conduction velocity from skin. After mapping, electroreceptive and mechanoreceptive fields were transferred onto acetate sheets.

Testing responsiveness to mechanical stimuli. An ascending series of von Frey probes was used to establish the mechanical threshold of each fiber. A fiber was classified as an MIA if the mechanical threshold was $\geq 6$ bar. This corresponds to fibers that did not respond to a 5.1 bar probe $(0.36$ $\mathrm{mm}$ diameter, $51 \mathrm{mN}$ ) but may have responded to the next higher $7.3 \mathrm{bar}$ probe $(0.51 \mathrm{~mm}$ diameter, $98 \mathrm{mN})$. As described previously (Meyer et al., 1991), 6 bars is 3 SDs above the mean threshold of C-fiber nociceptive afferents identified with standard mechanical search techniques. Mechanically insensitive afferents are not necessarily unresponsive to mechanical stimuli, some can be activated by stronger mechanical stimuli (e.g., stronger von Frey hairs, forceps pinch, or needle insertion). To avoid injuring the receptive field, intense mechanical stimuli were not routinely used.

Testing responsiveness to heat. To test the heat responsiveness of the afferent, contact-free heat stimuli were delivered to the skin using a radiometer feedback-controlled carbon dioxide laser (Meyer et al., 1976). Because the receptive fields to be tested were often bigger than the laser diameter $(8 \mathrm{~mm})$, the receptive field was divided into several, nonoverlapping test areas. After a given spot was tested, the laser was directed toward the next test point, and testing was repeated until the complete receptive field was studied.

All heat stimuli were started from a baseline temperature of $38^{\circ} \mathrm{C}$ that was held for at least $3 \mathrm{sec}$. To locate heat-sensitive spots, a $49^{\circ} \mathrm{C}$ stimulus of 1 or $3 \mathrm{sec}$ duration was delivered to the test area. If the afferent responded within $1 \mathrm{sec}$ of the stimulus onset, the afferent was classified as a type II afferent (Treede et al., 1998). Heat thresholds were determined at spots responsive to the initial stimulus after waiting at least 3 min to allow for recovery from possible fatigue (Treede et al., 1998). Thresholds were determined with $1 \mathrm{sec}$ duration stimuli that started from baseline $\left(38^{\circ} \mathrm{C}\right)$ and increased in $1^{\circ} \mathrm{C}$ increments until the final temperature of $49^{\circ} \mathrm{C}$ was reached. Stimuli were either presented in a discrete staircase paradigm or as an ascending series in which each step was started from baseline and stimuli were presented $30 \mathrm{sec}$ apart. Threshold was defined as the temperature that evoked the first action potential in the afferent under study.

As reported earlier (Treede et al., 1998), fibers with a high heat threshold to short-duration heat stimuli may respond to lower temperatures when the stimulus duration is increased. Afferents unresponsive to the initial, short-lasting $49^{\circ} \mathrm{C}$ stimulus were therefore tested with stimuli of $30 \mathrm{sec}$ duration and temperatures of 45,47 , and $49^{\circ} \mathrm{C}$. If such a stimulus induced a response, the afferent was classified as heat-insensitive with a type I response. In afferents unresponsive to a $49^{\circ} \mathrm{C}, 30 \mathrm{sec}$ stimulus, a $53^{\circ} \mathrm{C}$ stimulus of $30 \mathrm{sec}$ duration was finally delivered to the receptive field. To minimize tissue injury, prolonged stimuli were administered only at a single spot inside the electroreceptive and/or mechanoreceptive field. Two measures were taken to avoid effects that might compromise the response to capsaicin that followed the heat testing: (1) when feasible, the $53^{\circ} \mathrm{C}, 30 \mathrm{sec}$ stimulus was presented to an area different from the capsaicin injection site and (2) as with all stimuli of long duration (30 $\mathrm{sec}$ ), the stimulus was aborted when the afferent started to respond. Afferents not responding to the $53^{\circ} \mathrm{C}, 30 \mathrm{sec}$ stimulus were regarded as heat-unresponsive, high-threshold mechanoreceptors (HTMs) (Perl, 1968). Responsive afferents were classified according to the criteria reported previously (Treede et al., 1998). Briefly, a type II response was characterized by a rapid onset $(<1 \mathrm{sec})$, an early peak discharge $(<1 \mathrm{sec})$, and an adapting response during the stimulation. Afferents showing a late onset $(>0.2 \mathrm{sec})$, a late peak discharge rate $(>2 \mathrm{sec})$, and an increasing response during the stimulation were classified as heatinsensitive with a type I response. For stimuli that had been aborted, the late response was used to classify the afferents as type I afferents.

For this study, all fibers with a type II heat response were considered to be heat-sensitive afferents. All other fibers (including type I afferents) were considered to be heat-insensitive.

Capsaicin injections. Capsaicin was prepared in Tween 80 and saline as described earlier (LaMotte et al., 1991). In some experiments, Evans Blue $(0.5,1$, or $4 \%)$ was added to the capsaicin solution to delineate the spread of the injected volume. A standard dose of $10 \mu \mathrm{g}$ of capsaicin in a volume of $10 \mu \mathrm{l}$ was injected with a Becton Dickinson (Franklin Lakes, NJ) LoDose syringe. The area of spread was confined within the area covered by the heat stimulus, i.e., it was $<8 \mathrm{~mm}$ in diameter. When 
Table 1. Properties of afferents tested

\begin{tabular}{|c|c|c|c|c|c|}
\hline & \multirow{2}{*}{$\frac{\text { Heat-sensitive }}{\text { Type II }}$} & \multicolumn{2}{|c|}{ Heat-insensitive } & \multicolumn{2}{|c|}{ Mechanosensitivity } \\
\hline & & Type I & HTM & MSA & MIA \\
\hline$n(\text { total } 57)^{a}$ & 21 & 14 & $19^{b}$ & 27 & 30 \\
\hline Conduction velocity $(\mathrm{m} / \mathrm{sec})$ & $11.1 \pm 0.6$ & $18.8 \pm 2.7^{*}$ & $13.6 \pm 2.5$ & $18.0 \pm 2.1$ & $10.5 \pm 0.6^{* * *}$ \\
\hline \multicolumn{6}{|l|}{ Mechanical threshold (bar) } \\
\hline median & 11 & $3.7^{* *}$ & 8.2 & 3.5 & 13 \\
\hline Heat threshold $\left({ }^{\circ} \mathrm{C}\right)$ & & & No response to a & & \\
\hline Median & $48.0(13)$ & $>49(14)$ & $53^{\circ} \mathrm{C}, 30 \mathrm{sec}$ stim- & $>49(18)$ & $49(11)$ \\
\hline Mean & $47.4 \pm 0.7$ & & ulus & & $47.9 \pm 0.5$ \\
\hline \multicolumn{6}{|l|}{ Receptive field size $\left(\mathrm{mm}^{2}\right)$} \\
\hline Electroreceptive & $369 \pm 66(15)$ & $258 \pm 59(2)$ & $532 \pm 200(7)$ & $516 \pm 204(7)$ & $401 \pm 58(19)$ \\
\hline Mechanosensitive & $61 \pm 13(17)$ & $67 \pm 14(12)$ & $26 \pm 11(12)$ & $69 \pm 13(21)$ & $46 \pm 13(21)$ \\
\hline Tested with capsaicin & $16^{d}$ & 13 & 16 & $21^{c}$ & 25 \\
\hline
\end{tabular}

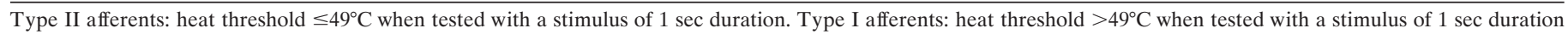

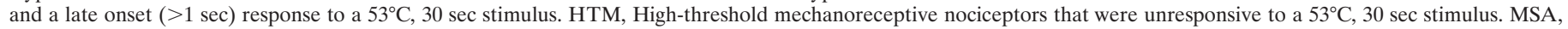

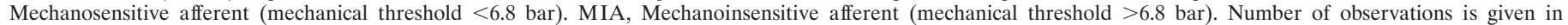
parentheses.

${ }^{a}$ Three of these afferents were not tested with heat stimuli.

${ }^{b}$ Eight afferents had a heat threshold $>49^{\circ} \mathrm{C}$ but were not tested with the $53^{\circ} \mathrm{C}, 30 \mathrm{sec}$ stimulus; these afferents are not type II but may be type I.

${ }^{c}$ One of these afferents was not tested for sensitivity to heat.

${ }^{d}$ All of the type II afferents tested with capsaicin had mechanical thresholds $>6$ bars.

*Significantly different from heat-sensitive (type II) fibers $(t$ test; $p<0.01)$.

**Significantly different from heat-sensitive (type II) fibers (Mann-Whitney $U$ test; $p<0.001$ ).

***Significantly different from MSAs $(t$ test; $p<0.01)$.

multiple injections were administered, the first injection was usually done $4 \mathrm{~mm}$ distal and outside of the electroreceptive and mechanoreceptive fields to check for remote effects of capsaicin. A second injection was performed inside an area that was purely electroreceptive. In afferents with a mechanoreceptive field, injections into this area were usually done last. Before injection of capsaicin, any ongoing activity in the fiber was recorded for $1 \mathrm{~min}$. Insertion of the needle was followed by a $10-15 \mathrm{sec}$ waiting period to ensure that insertion-related activity had decreased before the capsaicin injection. Responses to the injection of capsaicin were recorded for $10 \mathrm{~min}$ or until injection-related activity had ceased.

Data collection. Action potentials were displayed, recorded, and stored on a personal computer using a computer-based data acquisition board and a customized data acquisition and analysis software (DAPSYS; Brian Turnquist, Johns Hopkins University). DAPSYS allowed on- and off-line discrimination of different action potential waveforms based on multiple time-amplitude window criteria. In addition to the recording of action potential waveforms, DAPSYS controlled the laser that was used to apply heat stimuli to the skin, and it recorded the corresponding skin temperature before, during, and after such a stimulus. Furthermore, DAPSYS was used to time the different phases of the injection protocol by providing an auditory signal at the appropriate time points to start the needle insertion and the actual injection. Recorded action potentials and events (heat and mechanical stimuli) were time-stamped, which allowed the time course and neuronal activity to be directly related to the manipulations performed.

Statistical analysis. STATISTICA for Windows (1997; StatSoft, Tulsa, OK) was used for data analysis. Depending on the data to be analyzed, parametric and nonparametric procedures were used (Student's $t$ test, ANOVA, Wilcoxon, and Mann-Whitney $U$ test) when appropriate. Level of significance was $p<0.05$. Data are presented as mean \pm SEM.

\section{RESULTS}

A total of 57 A-nociceptors that innervated hairy skin were studied in 45 experiments. Conduction velocities, as measured from the nerve stimulation electrode, ranged from 4.7 to $53 \mathrm{~m} / \mathrm{sec}$ (mean, $14 \pm 1.2 \mathrm{~m} / \mathrm{sec}$ ). Fibers classified as type I heat-responsive had significantly higher conduction velocities $(18.8 \pm 2.7 \mathrm{~m} / \mathrm{sec}$; $n=14)$ than type II fibers $(11.1 \pm 0.6 \mathrm{~m} / \mathrm{sec} ; n=21 ; p<0.01$; $t$ test). Four of the nociceptors had conduction velocities $>30$ $\mathrm{m} / \mathrm{sec}$ and therefore would be considered to be $\mathrm{A} \beta$ fibers; three of these had a type I heat response, and one was an HTM. In addition, type I heat-responsive fibers had lower mechanical thresholds (median 3.7 bar) than type II heat-sensitive afferents (11 bar; $p<0.01$; Mann-Whitney $U$ test). These findings are consistent with a previous study (Treede et al., 1998). Four of the type II afferents had mechanical thresholds $<6$ bar, 10 type II fibers could be excited by von Frey hairs $>6$ bar, five type II afferents only responded to needle insertion or forceps squeeze, and two type II afferents did not respond to any mechanical stimulus. MIAs had significantly lower conduction velocities $(10.5 \pm 0.63 \mathrm{~m} / \mathrm{sec} ; n=30)$ than MSAs $(18.0 \pm 2.1 \mathrm{~m} / \mathrm{sec} ; n=27$; $p<0.01 ; t$ test). Table 1 summarizes some of the characteristic features of the fibers studied.

To compare the responses to capsaicin across different fiber populations, only the largest response to capsaicin (number of action potentials per $10 \mathrm{~min}$ ) in each fiber was used for analysis, regardless of the site of injection in the receptive field. In six fibers, capsaicin injection did not lead to excitation. Three of these afferents (all MIA, two unresponsive to a $53^{\circ} \mathrm{C}, 30 \mathrm{sec}$ stimulus, one with heat threshold $>49^{\circ} \mathrm{C}, 1 \mathrm{sec}$ ) were injected with capsaicin at multiple sites inside the electrical receptive field, but none of the administrations resulted in excitation. In the other three afferents (all MSA, two type I, one HTM), responsiveness to capsaicin was tested by a single injection into the receptive field.

\section{Type II heat-responsive A-fiber nociceptors exhibit a strong response to capsaicin}

A representative response to capsaicin obtained from a type II heat-sensitive nociceptor is illustrated in Figure 1 (left panel). Before the injection, the afferent was silent. Although all of the type II afferents that were injected with capsaicin were MIAs and had high mechanical thresholds, needle insertion into the receptive field induced a weak, short-lasting response in most of them 
A)
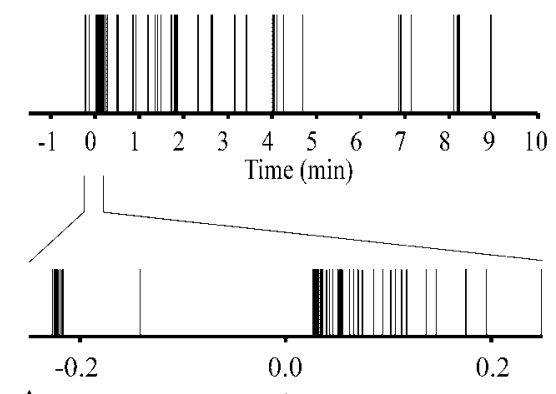

$\Delta$

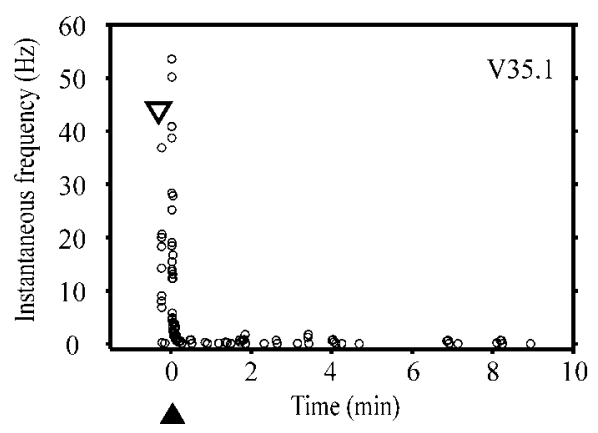

B) Type I A-fiber nociceptor
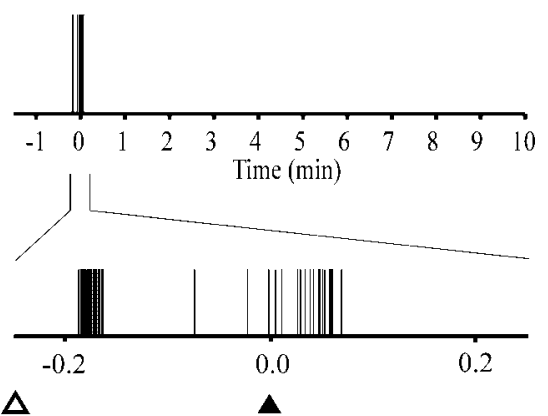

$\Delta$

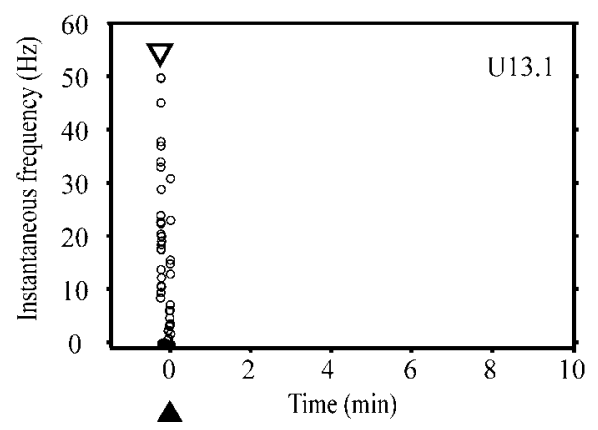

Figure 1. Specimen recordings of a response to capsaicin. $A$, Response to intradermal injection of capsaicin $(10 \mu \mathrm{g} / 10 \mu \mathrm{l})$ for a type II heatsensitive afferent (heat threshold, $49^{\circ} \mathrm{C}, 1 \mathrm{sec}$; mechanical threshold, 8.6 bar). The capsaicin was injected into an area responsive to von Frey hair stimulation. The needle insertion induced some activity in the fiber that was followed by a strong discharge after injection of capsaicin. Activity could be observed for $9 \mathrm{~min}$ after the injection. $B$, Response for a type I heatinsensitive afferent (heat threshold, $53^{\circ} \mathrm{C}, 4 \mathrm{sec}$; mechanical threshold, 4.6 bar). The capsaicin was injected into the mechanical receptive field. The needle insertion itself induced a strong activation. The excitation caused by the injection of capsaicin was short-lived $(<5 \mathrm{sec})$, and no further action potentials were observed for $10 \mathrm{~min}$ after the injection. Top panels, Time course of action potential activity. Every vertical line marks the time of occurrence of an action potential. Middle panels, Response to needle insertion and capsaicin injection illustrated on an expanded time scale. Bottom panels, Instantaneous frequency. Every circle represents the instantaneous frequency and time of occurrence of an action potential. Open triangles indicate the time point of tone from computer to prompt needle insertion. Filled triangles indicate the time of tone for capsaicin injection.
(10 of 16) as is evident by the example in the left panel of Figure 1. After injection of capsaicin, the afferent exhibited a strong response with a peak instantaneous discharge frequency of $53 \mathrm{~Hz}$. Although the instantaneous discharge frequency declined rapidly, ongoing activity at a lower discharge rate could be observed for 9 min after the injection.

The average time course of the response to an intradermal capsaicin injection obtained from 16 type II heat-sensitive fibers is summarized in Figure $2 A$. Insertion of the needle was accompanied by weak activation in 10 afferents, but injection of capsaicin induced a strong discharge that was significantly larger than the activation observed by needle insertion alone $(6.3 \pm 1.7 \mathrm{vs}$ $53 \pm 6$ action potentials per minute; repeated measures ANOVA followed by Scheffé test; $p<0.001)$. The discharge induced during the first minute after the injection was also significantly larger than the discharge observed thereafter $(p<0.001)$. As illustrated in Figure $3 A$, the most vigorous activation occurred during the first $15 \mathrm{sec}$ after the start of the injection. This injection-induced discharge was significantly larger than the activation caused by needle insertion alone ( $46 \pm 7$ vs $6 \pm 1.7$ action potentials per $15 \mathrm{sec} ; n=16 ; p<0.001$; paired $t$ test).

A histogram of the total response during the $10 \mathrm{~min}$ after the capsaicin injection is shown in Figure $4 A$. All type II fibers responded to capsaicin. The majority of fibers (13 of 16) responded with $>50$ action potentials in $10 \mathrm{~min}$, and only 3 of 16 responded with $<40$ action potentials in $10 \mathrm{~min}$. The average response in type II heat-sensitive nociceptive fibers was $69 \pm 7$ action potentials per $10 \mathrm{~min}$.

\section{Most heat-insensitive afferents are capsaicin-insensitive}

An example of the response of a heat-insensitive afferent to capsaicin is shown in Figure $1 B$ (right panel). This afferent was characterized as a type I fiber because it responded to heat only when exposed to a long-lasting $53^{\circ} \mathrm{C}$ stimulus. Before needle insertion, the afferent was silent. Insertion of the needle induced a strong response with an instantaneous frequency of $50 \mathrm{~Hz}$. In contrast, the peak discharge induced by the capsaicin injection $(30 \mathrm{~Hz})$ was less than that induced by needle insertion alone. Furthermore, the injection-induced discharge quickly dissipated within $5 \mathrm{sec}$, and no further action potentials were recorded for 10 min after the injection. In contrast to type II heat-sensitive afferents, the activation in heat-insensitive afferents during the first $15 \mathrm{sec}$ after the start of the capsaicin injection did not significantly differ from the activation caused by needle insertion alone (Fig. 3B).

Figure $4 B$ shows the distribution of the capsaicin responses obtained from fibers that did not show a type II heat response and that were therefore classified as heat-insensitive. Heat-insensitive fibers included type I afferents $(n=13)$, HTM afferents $(n=11)$, and those afferents that did not show a type II heat response but were insufficiently tested for subclassification as a type I or HTM $(n=5)$. The majority of fibers (22 of 29) classified as heatinsensitive showed only a weak response to capsaicin ( $<20$ action potentials per $10 \mathrm{~min}$ ) (Fig. $4 \mathrm{~B}$, dashed line).

\section{Some heat-insensitive afferents are capsaicin-sensitive}

As can be seen from Figure $4 B$, five of the heat-insensitive nociceptive afferents were vigorously excited by capsaicin $(>100$ action potentials per $10 \mathrm{~min}$ ). An example of a capsaicin response in such a fiber is given in Figure 5. This afferent did not respond to short-duration ( $1 \mathrm{sec}$ ) heat stimuli up to $49^{\circ} \mathrm{C}$ but responded to two applications of a $49^{\circ} \mathrm{C}, 30 \mathrm{sec}$ heat stimulus with latencies to the first action potential of 10 and $12 \mathrm{sec}$. This afferent was therefore classified as a type I afferent (mechanical threshold, 2.46 bar). Figure $5 A$ shows the response to injection of the vehicle into the mechanoreceptive field. The afferent responded with a few action potentials to needle insertion, but no response was seen during the $10 \mathrm{~min}$ after the injection of the vehicle. Figure $5 B$ illustrates the response after capsaicin injection into another part of the mechanoreceptive field. Again, the afferent responded with a few action potentials to the 

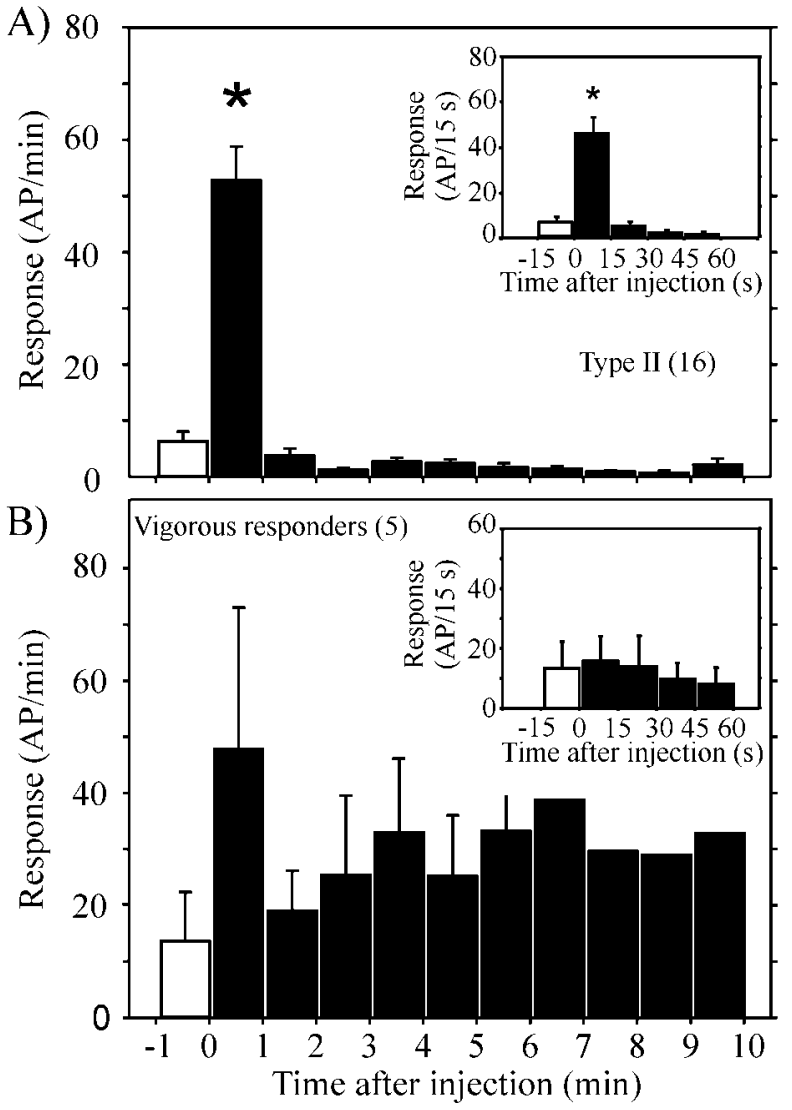

Figure 2. Average time course of capsaicin-evoked responses. A, Fibers with a type II heat response $(n=16)$. Type II fibers showed a strong response to capsaicin injection and only a weak response to needle insertion. $B$, Subset of heat-insensitive afferents with a vigorous response to capsaicin. These afferents $(n=5)$ were not type II heat-sensitive afferents but responded with $>100$ action potentials per 10 min to capsaicin injection. Open bars indicate the response to needle insertion, and filled bars represent the response to capsaicin (bin size $=1 \mathrm{~min}$ ). Inset, Expanded display at time of injection (bin size $=15 \mathrm{sec}$ ).
A)

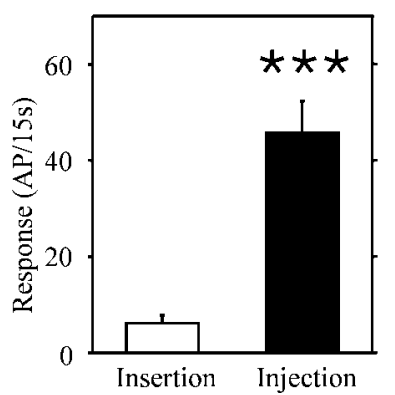

B)

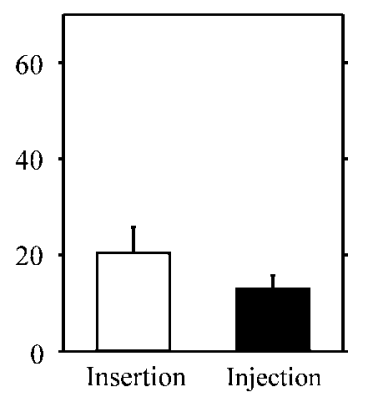

Figure 3. Comparison of the responses to needle insertion and the responses during the first $15 \mathrm{sec}$ after the capsaicin injection. A, Type II heat-sensitive afferents. The response after capsaicin injection was significantly larger than the response to needle insertion alone $(p<0.001$; paired $t$ test; $n=16$ ). $B$, Heat-insensitive afferents. In these afferents, capsaicin injection did not lead to a significantly larger response than needle insertion alone $(n=24)$. The five heat-insensitive afferents that did not respond to capsaicin are excluded.

needle insertion. In contrast to the injection of the vehicle, capsaicin injection was followed by a vigorous response, and this response lasted $>10 \mathrm{~min}$. Of the other four afferents that
A)

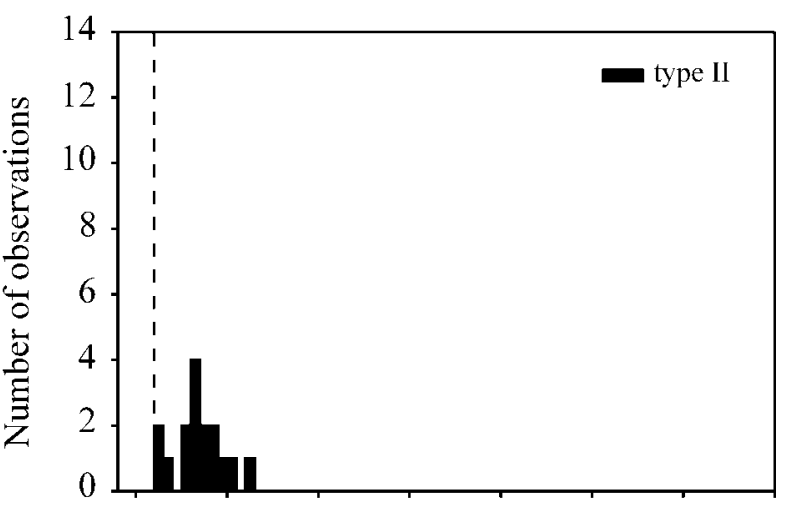

B)

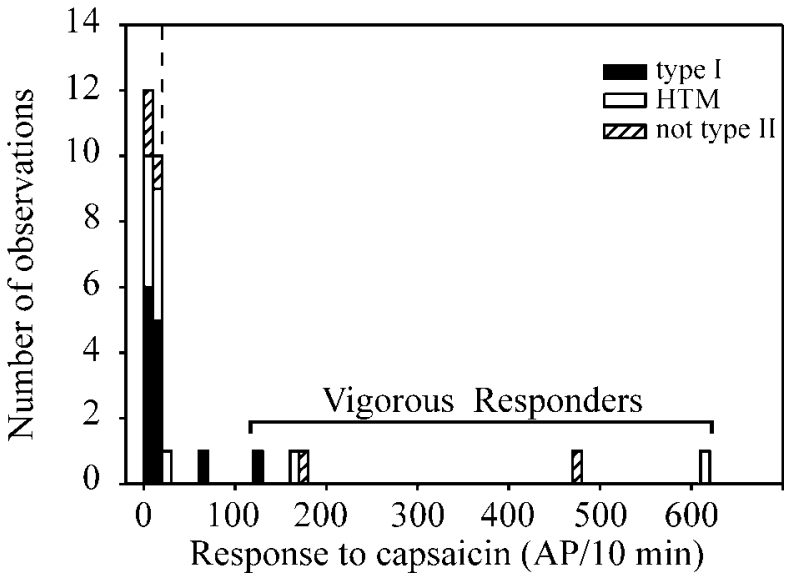

Figure 4. Distribution of the total response to capsaicin in $10 \mathrm{~min}$. A, Type II heat-sensitive afferents. All type II fibers gave a response to capsaicin that was $>20$ action potentials in $10 \mathrm{~min}$ (dashed line). In fact, the majority of fibers exhibited a strong response to capsaicin $(>50$ action potentials in $10 \mathrm{~min}) . B$, Heat-insensitive afferents. The majority of fibers categorized as HTM, type I, or not type II had very weak responses to capsaicin ( $\leq 20$ action potentials per $10 \mathrm{~min}$ ). However, five fibers not showing a type II response to heat showed the most vigorous responses to capsaicin ( $>100$ action potentials per $10 \mathrm{~min}$ ). Note: stacked histogram.

showed a vigorous response to capsaicin ( $>100$ action potentials per $10 \mathrm{~min}$ ), two afferents did not respond to a $53^{\circ} \mathrm{C}, 1 \mathrm{sec}$ thermal stimulus and thus were not type II afferents; another two afferents were unresponsive to the $53^{\circ} \mathrm{C}, 30 \mathrm{sec}$ stimulus and thus were HTMs.

Figure $2 B$ illustrates the time course of capsaicin-induced response for these five afferents insensitive to heat. In contrast to the time course of response seen in type II afferents (Fig. 2A), the response in these heat-insensitive afferents was long-lasting, i.e., the response did not markedly decrease during the 10 min observation period. Thus, responsiveness to capsaicin can exist in afferents that are insensitive to heat.

\section{Mechanically insensitive afferents exhibit a strong response to capsaicin}

The previous analysis separated the data into two populations based on heat sensitivity. Nociceptors may also be separated into MSAs and MIAs based on a mechanical threshold of 6 bar (Meyer et al., 1991; Treede et al., 1998).

Figure 1 also illustrates representative responses to capsaicin observed in a fiber classified as an MIA (panel A, left side) or an MSA (panel B, right side). The capsaicin-induced activity was 
A)
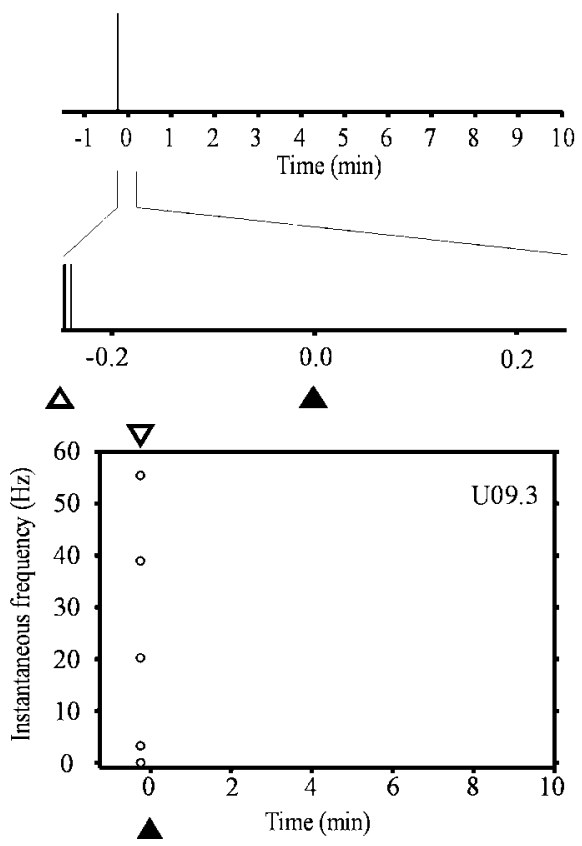

B)
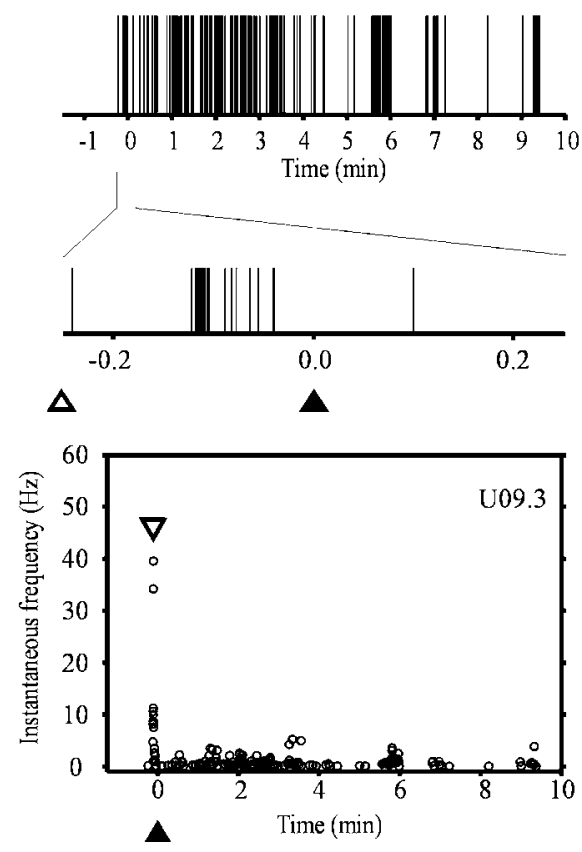

Figure 5. Vigorous response to capsaicin in a heat-insensitive afferent. $A$, Response to vehicle. $B$, Response to capsaicin. This afferent (mechanical threshold $=2.46$ bar) did not respond to short-duration heat stimuli $(1 \mathrm{sec})$ up to $49^{\circ} \mathrm{C}$. However, the afferent responded to a longlasting stimulus $\left(49^{\circ} \mathrm{C}, 30 \mathrm{sec}\right)$ and was therefore classified as a type I, heat-insensitive afferent. Both injections were performed inside the mechanosensitive receptive field, and the injection of vehicle preceded the injection of capsaicin. Insertion of the needle induced activity in the afferent for both injections. However, only the injection of capsaicin induced lasting activation of the afferent. Same format as Figure 1.

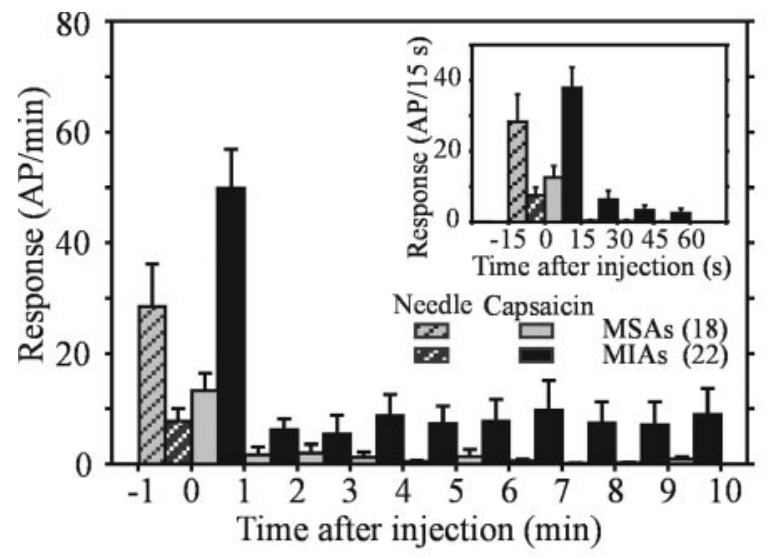

Figure 6. Time course of the capsaicin response in mechanically sensitive ( gray columns) and mechanically insensitive (black columns) afferents. In mechanically sensitive afferents $(n=18)$, needle insertion itself produced the largest response. In contrast, the largest discharge in mechanically insensitive afferents $(n=22)$ was observed during the first minute after injection of capsaicin. The response to capsaicin was significantly larger in mechanically insensitive than mechanically sensitive afferents $(p<0.001$; two-way ANOVA with repeated measures followed by multiple $t$ tests with Bonferroni correction). The six afferents (three MIAs, three MSAs) that did not respond at all to the capsaicin injection are excluded.

much more pronounced in the MIA than the MSA. Figure 6 illustrates the time course of the average responses obtained for the MIA and MSA populations based on the largest response observed in each afferent that gave a response to the capsaicin injection $(n=40)$. In MSAs, needle insertion itself induced a stronger discharge than the injection of capsaicin. In contrast, needle insertion induced only a weak discharge in MIAs that was followed by a significantly larger discharge after injection of capsaicin. Excitation during the first minute after the injection was significantly stronger in MIAs $(50 \pm 7$ action potentials per minute) than in MSAs ( $13 \pm 3$ action potentials per minute; $p<$ 0.001 ; Scheffé test). In both fiber populations, an ongoing dis-
A)

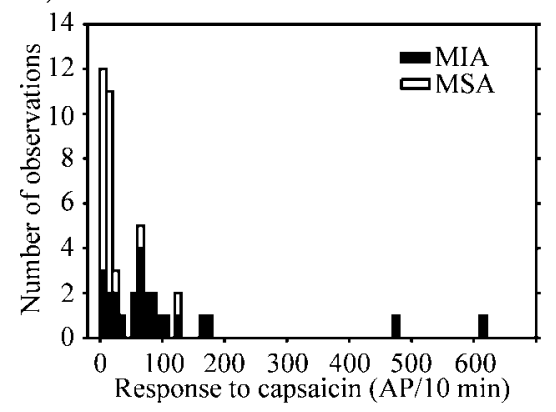

B)

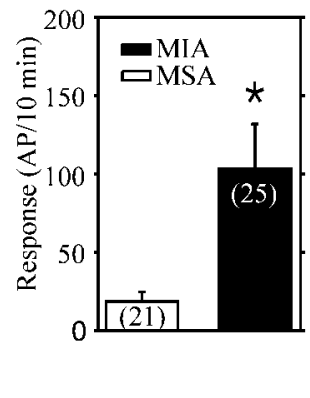

Figure 7. Comparison of capsaicin responses in mechanically sensitive and mechanically insensitive afferents. $A$, Histogram for the total number of action potentials observed in 10 min after the injection of capsaicin. Only the largest response to capsaicin obtained in each fiber was included $(n=46)$. Most of the vigorous responses ( $>50$ action potentials per 10 min) were observed in fibers classified as mechanically insensitive. $B$, Average response of the population during the $10 \mathrm{~min}$. Mechanically insensitive afferents exhibited a significantly greater response to capsaicin than mechanically sensitive afferents ( $p<0.05 ; t$ test). Numbers in parentheses give the number of fibers in each group.

charge could be observed for 10 min after the injection, and this discharge tended to be larger in MIAs.

The distribution of the total number of action potentials observed during the $10 \mathrm{~min}$ period after the injection appeared to have two peaks (Fig. $7 A$ ), i.e., weak responders ( $\leq 20$ action potentials per $10 \mathrm{~min})$ and strong responders $(>20$ action potentials/10 min). According to this criterion, 23 fibers were weak responders, and 23 were strong responders. All but two MSAs responded weakly. Most (17 of 19) of the fibers with high responses were MIAs (Fig. $7 A$ ). As illustrated in Figure $7 B$, the total response in MIAs (103 \pm 28 action potentials per $10 \mathrm{~min})$ was significantly larger than in MSAs $(18 \pm 6$ action potentials per $10 \mathrm{~min} ; p<0.02 ; t$ test $)$. These findings show that mechanically insensitive afferent nerve fibers are more sensitive to capsaicin than mechanically sensitive afferents. 


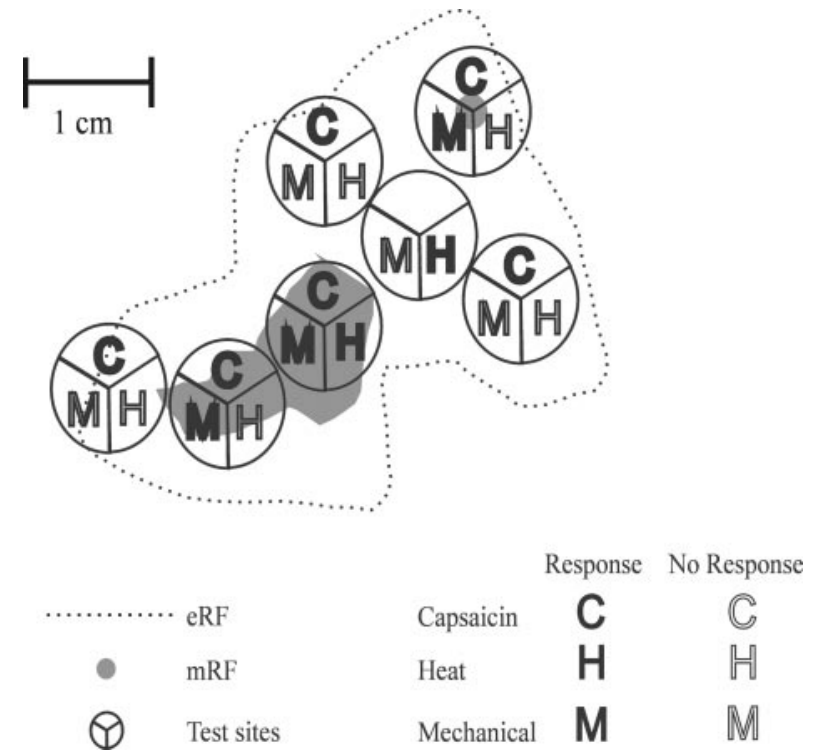

Figure 8. Responsiveness to different stimulus modalities varies across the receptive field of a type II fiber. The mechanosensitive receptive field (gray area) and the electroreceptive field (dotted line) are indicated. Multiple test sites indicated by the circles were tested with different stimulus modalities (heat, mechanical, capsaicin). The size of the circle corresponds to size of the laser beam used for the heat testing. Capsaicin induced a response at all injection sites, whereas only two test sites were heat-sensitive. Responses to needle insertion were observed in two locations inside the continuous mechanoreceptive field. This afferent was characterized as type II heat-sensitive with a heat threshold of $49^{\circ} \mathrm{C}, 1 \mathrm{sec}$ and a mechanical threshold of 11.2 bar.

\section{Responsiveness to different stimulus modalities is not equally distributed across the receptive field}

Figure 8 depicts an example in which multiple capsaicin injections were performed in the electroreceptive and mechanoreceptive fields of an afferent fiber. The circles indicate areas in which mechanical (15 bar von Frey), heat $\left(49^{\circ} \mathrm{C}, 1 \mathrm{sec}\right)$, and/or capsaicin $(10 \mu \mathrm{g} / 10 \mu \mathrm{l})$ testing was performed. Only two areas responded to heat. One area that was located in a purely electroreceptive part responded to a $49^{\circ} \mathrm{C}, 1 \mathrm{sec}$ heat stimulus, and the fiber was therefore classified as type II. At the second site, which was located in the mechanoreceptive field, a type II-like heat response was obtained to a $53^{\circ} \mathrm{C}, 30 \mathrm{sec}$ stimulus. In contrast to the heat stimuli, capsaicin excited the afferent at all injection sites, including those unresponsive to short duration heat stimuli. Capsaicininduced activity ranged from 20 to 93 action potentials in $10 \mathrm{~min}$, and the highest response was observed in the part of the mechanoreceptive field unresponsive to the $49^{\circ} \mathrm{C}, 1 \mathrm{sec}$ heat stimulus.

Figure 9 summarizes the incidence of mechanical, heat, and capsaicin sensitivity for sites in type II fibers tested with all three stimulus modalities. Most of the test sites (24 of 36) responded to only one (12) or two (12) modalities. Only eight sites were sensitive to all three stimulus modalities. The majority of test sites (29 of 36) were chemosensitive. Eleven test sites were sensitive to capsaicin, but not to heat or mechanical stimuli. In the other 10 sites, sensitivity to capsaicin was present together with sensitivity to only one other stimulus modality. These findings argue that different areas inside the receptive field of polymodal nociceptors vary in their sensitivity to mechanical, thermal, and chemical stimuli.

More than one capsaicin injection into the electroreceptive and/or mechanoreceptive field was administered for 28 fibers in

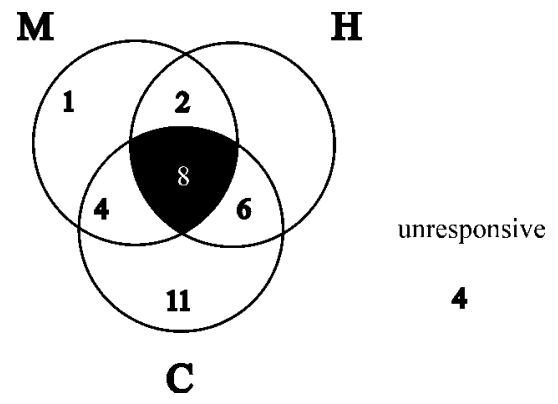

Figure 9. Polymodality of different sites within the receptive field of type II afferents. Venn diagram for the different stimulus modalities to which a test site inside the electroreceptive field of a type II fiber was sensitive. Only sites tested with all three modalities are included. As for Figure 8, a test site was classified as mechanosensitive when it responded to von Frey hair stimulation. Most test sites were responsive to capsaicin (29 of 32), and many of these were sensitive to capsaicin only (11 of 29). Only eight capsaicin-sensitive sites were also sensitive to heat and mechanical stimuli. Four sites were insensitive to each of the stimulus modalities. Data come from 15 type II afferents.

this study. Repetitive injections into electroreceptive areas of the receptive field yielded a wide range of responses that were not significantly different from each other. In addition, multiple injections into the mechanosensitive receptive field resulted in similar response magnitudes. For nine fibers, an injection was made into an electroreceptive and a mechanosensitive area of the receptive field. Five of these afferents were MIAs, and four were MSAs. The responses from the mechanosensitive areas $(37 \pm 11$ action potentials per $10 \mathrm{~min}$ ) were significantly larger than those from purely electroreceptive parts of the receptive field (13 \pm 8 action potentials per $10 \mathrm{~min} ; p<0.05$; paired $t$ test).

Eleven capsaicin injections were made outside the electroreceptive field. Eight of these resulted in no response. For the three injections that resulted in a weak response $(<20$ action potentials in $10 \mathrm{~min}$ ), the Evans Blue had clearly spread into the receptive field. This provides evidence that the electroreceptive field mapping procedure delineated the border of the receptive field.

\section{DISCUSSION}

The results of this study provide evidence for the existence of capsaicin-sensitive vanilloid receptors in small myelinated nociceptive afferents of the primate. Most capsaicin-sensitive afferents were responsive to heat but insensitive to mechanical stimuli. However, heat sensitivity was not a prerequisite for responsiveness to capsaicin, because vigorous excitation by capsaicin was observed in some heat-insensitive afferents. Moreover, within the receptive field of a given heat-sensitive A-fiber nociceptor, regions responsive to heat did not always correspond to regions responsive to capsaicin.

\section{Capsaicin activates heat-sensitive type II A-fiber nociceptors}

In this study all cutaneous type II heat-sensitive A-fiber nociceptors (16 of 16) were excited by capsaicin, providing for the first time direct evidence that vanilloid receptors are present in the axonal membrane of these cutaneous afferents. Earlier psychophysical experiments provided indirect evidence that capsaicin sensitivity may exist in A-fiber nociceptors. Type II A-fiber nociceptors in human skin mediate the first pricking sensation to stepped heat stimuli (Price et al., 1977; Campbell and LaMotte, 1983; Gronroos et al., 1996). The loss of the first heat pain and the corresponding evoked potential after repetitive topical capsaicin 
treatment suggest that type II A-fiber nociceptors become desensitized by capsaicin and therefore are indeed capsaicin-sensitive (Beydoun et al., 1996; Gronroos et al., 1996). In addition, the administration of a capsaicin analog produced heat analgesia in human skin (Davis et al., 1995a,b), and administration of capsaicin resulted in a dramatic increase in the latency for the detection of heat pain (Nolano et al., 1999).

Of the vanilloid receptors identified so far, only one has been reported to be responsive to capsaicin. The VR1 is responsive to heat and to capsaicin, protons, and resiniferatoxin (Caterina et al., 1997; Tominaga et al., 1998). A splice variant of VR1, VR.5'sv, is completely unresponsive to these stimuli (Schumacher et al., 2000). Furthermore, the VRL1 only responds to high-intensity heat stimuli but not to chemical stimuli such as capsaicin, resiniferatoxin, and protons (Caterina et al., 1999). The VR1 appears to be the critical transduction protein that mediates responses to capsaicin because afferent neurons from VR1-null mutant mice do not respond to capsaicin, and null mutant animals do not display pain behavior after capsaicin injection (Caterina et al., 2000). This suggests that only VR1 could account for the capsaicin sensitivity in heat-sensitive A-fiber nociceptors. In previous studies in rat, however, VR1 has only been localized in small-diameter neurons that are connected to small unmyelinated fibers in the periphery (Caterina et al., 1997). Two explanations may explain this apparent discrepancy: (1) in primate, the expression of VR1 may not be limited to small-diameter neurons but may also extend to medium- and large-diameter neurons. (2) Responses to capsaicin in A-fiber nociceptors may be mediated by yet unidentified vanilloid receptor splice variants of VR1 other than VR.5'sv.

\section{Capsaicin sensitivity without heat sensitivity}

One surprising finding of the present study was that some A-fiber nociceptors were insensitive to heat but responsive to capsaicin. In fact, the highest responses to capsaicin were observed in these afferents. These A-fiber nociceptors were also insensitive to mechanical stimuli. Thus, some A-fiber nociceptors may be considered to be cutaneous chemoreceptors. In support of this idea, A-fiber nociceptors were also found to give a more vigorous response to intradermal injection of a cocktail of inflammatory mediators than did C-fiber nociceptors (Davis et al., 1993).

Capsaicin sensitivity in heat-insensitive dorsal root ganglion cells has previously been reported (Kirschstein et al., 1997, 1999). As in the present study, however, the prevalence of capsaicin sensitivity in heat-insensitive afferent neurons was low ( 2 of 5 and 2 of 14). In contrast, recordings from dorsal root ganglion cells in rat demonstrated that sensitivity to capsaicin is restricted to neurons with a low heat threshold (Nagy and Rang, 1999a). It could be argued that the capsaicin responses in heat-insensitive afferents reported here are attributable to an unspecified capsaicin effect. This is unlikely, however, because a strong excitation by capsaicin was primarily observed in mechanically insensitive afferents (Fig. 6), whereas excitation caused by an unspecified effect should have been observed across different classes of afferents. Patch-clamp experiments on isolated membrane patches have recently shown that ion channels may be exclusively activated by either capsaicin or heat. Surprisingly, sensitivity to only one stimulus modality was more frequently observed than sensitivity to both (Nagy and Rang, 1999b). Therefore, the VR1 may be present in different molecular configurations that differ in their sensitivity to heat and capsaicin (Nagy and Rang, 1999b). It is plausible that such a segregation may be present in the entire axonal membrane of a neuron.

\section{Most type I afferents are not responsive to capsaicin}

The VRL1 is activated by high heat intensities but not capsaicin (Caterina et al., 1999). In rat, the VRL1, and not the VR1, receptor is found in medium- to large-diameter neurons. Our finding that most (11 of 13) type I A-fiber nociceptors did not respond to capsaicin is consistent with the hypothesis that the VRL1 receptor is responsible for heat transduction in this class of afferents.

\section{Role of capsaicin-sensitive A-fiber nociceptors}

Intradermal injection of capsaicin in humans produces intense pain and leads to secondary hyperalgesia to mechanical stimuli (LaMotte et al., 1991; Ali et al., 1996; Huang et al., 2000). Pain and hyperalgesia develop in the presence of an A-fiber block and thus are thought to be attributable mainly to activation of $\mathrm{C}$-fiber nociceptors (Ziegler et al., 1999). However, the A-fiber block experiments do not rule out a role for the A-fiber nociceptors because many A-nociceptors have very long unmyelinated branches that may extend beyond a radial nerve compression block (Peng et al., 1999).

\section{Mechanically insensitive afferents are excited by capsaicin}

The results of this study also demonstrate that afferents insensitive to mechanical stimuli are sensitive to capsaicin. In contrast to mechanically sensitive afferents, mechanically insensitive afferents exhibit a strong response to the capsaicin injection but only a weak response to needle insertion. Almost all responses to capsaicin in this study were obtained in mechanically insensitive afferents. In conjunction with the observation that type II heatsensitive fibers are capsaicin-sensitive, this finding is not surprising but consistent with an earlier study showing that type II heat-sensitive fibers are in fact insensitive to mechanical stimuli (Treede et al., 1998). Interestingly, a similar finding has been reported from microneurography experiments in humans showing that unmyelinated $\mathrm{C}$-fibers insensitive to mechanical stimuli are vigorously activated by capsaicin (Schmelz et al., 2000). The fact that capsaicin-sensitive fibers are insensitive to mechanical stimuli (including gentle pinching the skin) may explain why previous studies that used mechanical search techniques to locate receptive fields failed to identify these capsaicin responders (Szolcsanyi et al., 1988; Baumann et al., 1991). In the present study, a previously described electrical search technique (Meyer et al., 1991) was used to locate receptive fields.

\section{Sensitivity to different stimulus modalities does not coincide within the receptive field}

Most of the test sites (24 of 36) in type II heat-sensitive afferents responded to only one or two stimulus modalities. Sensitivity to all three modalities was observed at only eight test sites. This finding suggests that A-fiber nociceptors are not homogeneously responsive to different stimulus modalities across their innervation territory.

In a previous study, we reported that mechanical and heat sensitivity were colocalized for unmyelinated nociceptive fibers (Treede et al., 1990). However, in that study only unmyelinated fibers were investigated, and receptive fields were not mapped with electrical stimuli. Therefore, heat-sensitive but mechanoinsensitive sites might have been overlooked. In fact, a mismatch 
between spots sensitive to heat and mechanical stimuli has been reported for nociceptive C-fibers in humans (Olausson, 1998).

Sensitivity to a given stimulus ultimately depends on the presence of functional transduction proteins in the axonal membranes of the peripheral endings. Most likely, the transduction proteins responsive to different stimulus modalities are randomly distributed along the axon and its peripheral terminals. Sensitivity to a certain stimulus, however, may depend on the density of functional channels in the membrane, and this density may vary along the peripheral arborization of a given fiber.

\section{REFERENCES}

Ali Z, Meyer RA, Campbell JN (1996) Secondary hyperalgesia to mechanical but not heat stimuli following a capsaicin injection in hairy skin. Pain 68:401-411.

Baumann TK, Simone DA, Shain CN, LaMotte RH (1991) Neurogenic hyperalgesia: the search for the primary cutaneous afferent fibers that contribute to capsaicin-induced pain and hyperalgesia. J Neurophysiol 66:212-227.

Beydoun A, Dyke DBS, Morrow TJ, Casey KL (1996) Topical capsaicin selectively attenuates heat pain and A $\delta$ fiber-mediated laser-evoked potentials. Pain 65:189-196.

Campbell JN, LaMotte RH (1983) Latency to detection of first pain. Brain Res 266:203-208.

Campbell JN, Meyer RA (1983) Sensitization of unmyelinated nociceptive afferents in the monkey varies with skin type. J Neurophysiol 49:98-110.

Caterina MJ, Schumacher MA, Tominaga M, Rosen TA, Levine JD, Julius D (1997) The capsaicin receptor: a heat-activated ion channel in the pain pathway. Nature 389:816-824

Caterina MJ, Rosen TA, Tominaga M, Brake AJ, Julius D (1999) A capsaicin-receptor homologue with a high threshold for noxious heat. Nature 398:436-441.

Caterina MJ, Leffler A, Malmberg AB, Martin WJ, Trafton J, PetersenZeitz KR, Koltzenburg M, Basbaum AI, Julius D (2000) Impaired nociception and pain sensation in mice lacking the capsaicin receptor. Science 288:306-313.

Davis KD, Meyer RA, Campbell JN (1993) Chemosensitivity and sensitization of nociceptive afferents that innervate the hairy skin of monkey. J Neurophysiol 69:1071-1081.

Davis KD, Meyer RA, Turnquist JL, Filloon TG, Pappagallo M, Campbell JN (1995a) Cutaneous injection of the capsaicin analogue, NE21610 , produces analgesia to heat but not to mechanical stimuli in man. Pain 61:17-26.

Davis KD, Meyer RA, Turnquist JL, Filloon TG, Pappagallo M, Campbell JN (1995b) Cutaneous pretreatment with the capsaicin analog NE-21610 prevents the pain to a burn and subsequent hyperalgesia. Pain 62:373-378.

Gronroos M, Reunala T, Pertovaara A (1996) Influence of selective nerve fiber blocks on argon laser-induced thermal pain in the human skin. Neurosci Lett 211:143-145.

Huang JH, Ali Z, Travison TG, Campbell JN, Meyer RA (2000) Spatial mapping of the zone of secondary hyperalgesia reveals a gradual decline of pain with distance but sharp borders. Pain 86:33-42.

Kirschstein T, Busselberg D, Treede RD (1997) Coexpression of heat- evoked and capsaicin-evoked inward currents in acutely dissociated rat dorsal root ganglion neurons. Neurosci Lett 231:33-36.

Kirschstein T, Greffrath W, Busselberg D, Treede RD (1999) Inhibition of rapid heat responses in nociceptive primary sensory neurons of rats by vanilloid receptor antagonists. J Neurophysiol 82:2853-2860.

LaMotte RH, Shain CN, Simone DA, Tsai E-FP (1991) Neurogenic hyperalgesia: Psychophysical studies of underlying mechanisms. J Neurophysiol 66:190-211.

Meyer RA, Walker RE, Mountcastle VB (1976) A laser stimulator for the study of cutaneous thermal pain sensation. IEEE Trans Biomed Eng 23:54-60.

Meyer RA, Davis KD, Cohen RH, Treede R-D, Campbell JN (1991) Mechanically insensitive afferents (MIAs) in cutaneous nerves of monkey. Brain Res 561:252-261.

Nagy I, Rang H (1999a) Noxious heat activates all capsaicin-sensitive and also a subpopulation of capsaicin-insensitive dorsal root ganglion neurons. Neuroscience 88:995-997.

Nagy I, Rang HP (1999b) Similarities and differences between the responses of rat sensory neurons to noxious heat and capsaicin. J Neurosci 19:10647-10655.

Nolano M, Simone DA, Wendelschafer-Crabb G, Johnson T, Hazen E, Kennedy WR (1999) Topical capsaicin in humans: parallel loss of epidermal nerve fibers and pain sensation. Pain 81:135-145.

Olausson B (1998) Recordings of polymodal single C-fiber nociceptive afferents following mechanical and argon-laser heat stimulation of human skin. Exp Brain Res 122:44-54.

Peng YB, Ringkamp M, Campbell JN, Meyer RA (1999) Electrophysiological assessment of the cutaneous arborization of A-delta-fiber nociceptors. J Neurophysiol 82:1164-1177.

Perl ER (1968) Myelinated afferent fibres innervating the primate skin and their response to noxious stimuli. J Physiol (Lond) 197:593-615.

Price DD, Hu JW, Dubner R, Gracely RH (1977) Peripheral suppression of first pain and central summation of second pain evoked by noxious heat pulses. Pain 3:57-68

Schmelz M, Schmidt R, Handwerker HO, Torebjörk HE (2000) Encoding of burning pain from capsaicin-treated human skin in two categories of unmyelinated nerve fibres. Brain 123(Pt 3):560-571.

Schumacher MA, Moff I, Sudanagunta SP, Levine JD (2000) Molecular cloning of an N-terminal splice variant of the capsaicin receptor. Loss of N-terminal domain suggests functional divergence among capsaicin receptor subtypes. J Biol Chem 275:2756-2762.

Szolcsanyi J, Anton F, Reeh PW, Handwerker H (1988) Selective excitation by capsaicin of mechano-heat sensitive nociceptors in rat skin. Brain Res 446:262-268.

Tominaga M, Caterina MJ, Malmberg AB, Rosen TA, Gilbert H, Skinner K, Raumann BE, Basbaum AI, Julius D (1998) The cloned capsaicin receptor integrates multiple pain-producing stimuli. Neuron 21:531-543.

Treede R-D, Meyer RA, Campbell JN (1990) Comparison of heat and mechanical receptive fields of cutaneous $\mathrm{C}$-fiber nociceptors in monkey. J Neurophysiol 64:1502-1513.

Treede RD, Meyer RA, Raja SN, Campbell JN (1995) Evidence for two different heat transduction mechanisms in nociceptive primary afferents innervating monkey skin. J Physiology 483:747-758.

Treede R-D, Campbell JN, Meyer RA (1998) Myelinated mechanicallyinsensitive afferents from monkey hairy skin: heat response properties. J Neurophysiol 80:1082-1093.

Ziegler EA, Magerl W, Meyer RA, Treede RD (1999) Secondary hyperalgesia to punctate mechanical stimuli. Central sensitization to A-fibre nociceptor input. Brain 122(Pt 12):2245-2257. 